\title{
Inhibition of Rho-associated protein kinase increases the ratio of formation of blastocysts from single human blastomeres
}

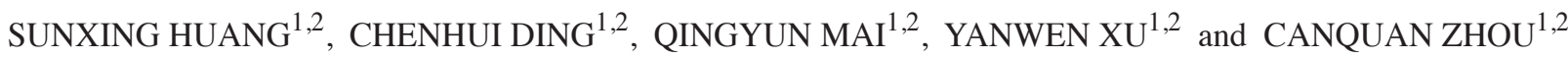 \\ ${ }^{1}$ Reproductive Medicine Center, First Affiliated Hospital of Sun Yat-sen University; \\ ${ }^{2}$ Laboratory of Reproductive Medicine of Guangdong, Guangzhou, Guangdong 510080, P.R. China
}

Received December 27, 2014; Accepted September 25, 2015

DOI: $10.3892 / \mathrm{mmr} .2016 .4766$

\begin{abstract}
Y-27632 is a specific inhibitor of Rho-associated protein kinases (ROCKs), which are downstream effectors of Rho GTPase. The present study aimed to determine the effect of the specific ROCK inhibitor, Y-27632, on fresh human embryos and on single blastomeres obtained from discarded human embryos. A total of 784 poor-quality embryos were included, of which 526 were allocated to blastocyst culture directly and the remaining 258 were allocated to blastomere isolation. Embryos and single blastomeres were cultured either with, or without, Y-27632. Embryonic development was observed and recorded daily from day 5 onwards. Y-27632 did not affect the ratio of blastocyst formation or the quality of the human embryos. The duration of blastocyst formation was compared between the two groups in the embryo culture. On day 5 , the blastocyst formation ratio in the experimental group was $11.4 \%(26 / 228)$, which was significantly $(\mathrm{P}=0.015)$ lower than the corresponding rate $(19.7 \% ; 44 / 223)$ in the control group. Survival analysis of the blastocyst formation duration showed that the median formation duration in the experimental group was significantly higher than that of the control group. The present study also obtained 1,192 blastomeres from 258 discarded day 3 embryos, and sibling blastomeres of similar sizes were equally allocated to experimental and control groups ( $n=596$ in each). Treatment with Y-27632 increased the blastocyst formation ratio of human individual blastomeres, with 82 blastocysts of 596 blastomeres (13.8\%), and 51 blastocysts of 596 blastomeres (8.6\%) formed in the presence and absence of $\mathrm{Y}-27632$, respectively $(\mathrm{P}=0.004)$. Compared with the control group, the mRNA and protein expression levels of E-cadherin in the blastocysts from blastomeres were enhanced by $\mathrm{Y}-27632(\mathrm{P}=0.022)$. In conclusion, the present study demonstrated that Y-27632 has different effects
\end{abstract}

Correspondence to: Professor Canquan Zhou, Reproductive Medicine Center, First Affiliated Hospital of Sun Yat-sen University, 58 Zhongshan Road II, Guangzhou, Guangdong 510080, P.R. China E-mail: zhoucanquan@hotmail.com

Key words: in vitro fertilization and embryo transfer, Y-27632, single blastomeres, Rho-associated protein kinase, E-cadherin on the cleavage-stage of embryos and single blastomeres. Y-27632 increases the ratio of formation of blastocysts from single human blastomeres, but inhibits the direct formation of blastocysts from discarded human embryos.

\section{Introduction}

In vitro fertilization and embryo transfer (IVF-ET) has become the most effective treatment for infertile couples in the past three decades (1). Embryo quality is one of the most important factors affecting the results of IVF-ET. Several studies have attempted to improve the quality of embryos cultured in vitro by determining the potential mechanism of embryonic development and adjusting the in vitro culture conditions (2-5). A zygote undergoes a series of cleavages and subsequently enters the blastocyst stage. Numerous physiological cellular processes, including apoptosis, cell-cell adhesion, cell polarity differentiation and proliferation, occur between the zygote and the blastocyst stages, and are vital for early embryonic development $(6,7)$.

Rho-associated protein kinase (ROCK)-LIM domain kinase 1/2-cofilin-actin is one of the most important signaling pathways regulating actin assembly and actin depolymerization. Previous studies $(8,9)$ have demonstrated the importance of actin filaments for oocyte and embryo development. Y-27632 is a specific inhibitor of ROCKs, which are downstream effectors of Rho GTPase that function in numerous physiological cellular processes, including contraction, adhesion, migration and proliferation (10). Y-2763 has been shown to have different effects on mammalian embryonic development. For example, Y-27632 has been found to inhibit mouse blastocyst cavity formation (11) and prevent early mouse embryo development (12). However, Y-27632 has also been found to stimulate the revivability of in vitro-produced bovine blastocysts following vitrification (13). In another study, Y-27632 was demonstrated to increase the post-thaw survival rates of embryos (14). Until recently, the precise effect of Y-27632 on human embryos and single blastomeres, remained to be fully elucidated (8,15-17).

Previous experiments have shown that single blastomeres from human cleavage-stage embryos are pluripotent and can differentiate into human embryonic stem cells (hESCs) $(18,19)$. Several studies have found that Y-27632 markedly reduces the apoptosis of dissociative hESCs and improves the recovery of 
hESCs from cryopreservation (20-23). It has also been shown to effectively improve the survival of other pluripotent cell, including induced pluripotent stem (iPS) cells (24). These findings indicate the utility of Y-27632 in a variety of applications across a number of different cell types $(22,25,26)$. However, the direct effect of Y-27632 alone on single blastomeres from cleavage-stage embryos remains to be elucidated.

Therefore, the present study aimed to determine the effect of Y-27632 on the development of fresh human embryos and single blastomeres obtained from human embryos. In order to overcome the ethical problems associated with experimentation on human embryos, poor-quality embryos and embryos in which abnormal fertilization occurred were used.

\section{Materials and methods}

Ethical statement. Ethical approval for the present study was granted by the Institutional Ethics Committee of the First Affiliated Hospital of Sun Yat-sen University (Guangzhou, China). Couples agreed to donate their discarded embryos for the present study, following being clearly informed about the details, and written informed consent was obtained from these couples.

Patients. All the discarded embryos were donated for use in the present study by patients undergoing fresh in vitro fertilization (IVF)/intracytoplasmic sperm injection cycles at the Reproductive Medical Center, The First Affiliated Hospital of Sun Yet-sen University. Between March 2010 and May 2010, and between November 2010 and April 2011, a total of 224 couples agreed to donate their discarded embryos for the present study following being clearly informed of the details. A total of 784 embryos were donated by these patients, of which 526 were allocated to the first part of the present study, and the remaining 258 were allocated to the second part. The experimental protocol was approved of the First Affiliated Hospital of Sun Yat-sen University.

All the patients received a long protocol for stimulation. Gonadotropin with follicle-stimulating hormone (FSH; Gonal-F; Merck-Serono Company, Corsier-sur-Vevey, Switzerland) was administered at 150-300 IU/day between days 3 and 5. The patient received 10,000 IU human chorionic gonadotropin (hCG; Livzon Pharmaceutical Group Co., Ltd., Guangdong, China), and transvaginal ultrasound-guided oocyte retrieval was performed $36 \mathrm{~h}$ later (Aloka SSD-3500; Hitachi-Aloka Medical, Ltd., Tokyo, Japan). All oocytes were inseminated $3 \mathrm{~h}$ following retrieval.

Day 3 embryo assessment. The day 3 embryos were graded, as described previously (27) using an Olympus IX71 microscope (Olympus Corp, Tokyo, Japan). Briefly, grade 1, equal-sized symmetrical blastomeres; grade 2, uneven blastomeres with $<10 \%$ fragmentation; grade 3, 10-50\% fragmentation; grade 4 $>50 \%$ fragmentation. Grade 3 or 4 embryos, or embryos with fewer than four blastomeres were considered to be of poor quality, and those embryos demonstrating poor-quality were used for the present study.

Blastomere isolation. In the second part of the experiment, 258 of the collected day 3 embryos were used. Blastomeres from the discarded embryos were isolated by micromanipulation. Briefly, the zona pellucida was removed with $0.5 \%$ pronase (Sigma-Aldrich, St. Louis, MO, USA), and the embryos were washed twice in calcium- and magnesium-free phosphate-buffered solution (PBS). Single blastomeres were isolated with a $50-\mu \mathrm{m}$ diameter micropipette by gentle repeated aspiration.

Blastocyst formation. Following morphological assessment of the day 3 embryos, for the first part of the experiment, 526 embryos were collected and allocated to blastocyst culture with or without Y27632 (Sigma-Aldrich). In the second part of the experiment, following isolation of the blastomeres, sibling blastomeres of similar size, derived from the same embryo, were paired and allocated to blastocyst culture, with or without, Y-27632. The remaining unpaired blastomeres were abandoned.

The embryos and single blastomeres were cultured in conventional culture medium comprised of blastocyst medium (Sage) supplemented with $10 \%$ serum protein substitute (Sage) for an additional 5 days at $37^{\circ} \mathrm{C}$ and $5 \% \mathrm{CO}_{2}$. In the experimental group, $10 \mu \mathrm{M}$ Y-27632 was added to the blastocyst culture medium. The concentration of $10 \mu \mathrm{M}$ has been a commonly used working concentration in previous human embryo and hESC studies (20-26).

From day 5, embryonic development was recorded daily, including the time of blastocyst formation and the blastocyst grade, as described by Gardner et al (28). The development of single blastomeres was also recorded daily from day 5 onwards (Fig. 1).

Immunofluorescence. On day 6, a number of the blastocysts from the single blastomeres were stained with $2 \mu \mathrm{g} / \mathrm{ml}$ Hoechst-33342 (Sigma-Aldrich) to count the numbers of cells. On day 7, immunofluorescence analysis was performed to detect the expression levels of the pluripotency marker octamer-binding transcription factor (Oct)3/4 and E-cadherin in the blastocysts cultured from single blastomeres. Blastocysts were fixed with $4 \%$ formaldehyde at room temperature (RT) and permeabilized with $0.2 \%$ Triton-X100 (Sigma-Aldrich) in PBS for $20 \mathrm{~min}$, prior to blocking with $10 \%$ goat serum (Wuhan Boster Biological Technology, Ltd., Wuhan, China) in PBS for $1 \mathrm{~h}$. Subsequently, the blastocysts were incubated with the following primary antibodies overnight at $4^{\circ} \mathrm{C}$ : Polyclonal rabbit anti-human OCT3/4 (1:200; 18976; Abcam, Cambridge, UK) and polyclonal rabbit anti-human E-cadherin antibody $(1: 100$; 15148; Abcam). The blastocysts were then incubated with a fluorescent-conjugated secondary antibody (goat anti-rabbit IgG fluorescein isothiocyanate; $1: 40 ; 6717$; Abcam) for $1 \mathrm{~h}$ at RT. The cell nuclei were stained with 4',6-diamidino-2-phenylindole (Sigma-Aldrich). Specimens were observed using Zeiss LSM 510 Meta Confocal Microscope (Carl Zeiss AG, Oberkochen, Germany).

Reverse transcription-quantitative polymerase chain reaction (RT-qPCR) to measure the mRNA expression levels of E-cadherin. Total RNA was extracted from blastocysts (20 blastocysts per group) on day 7 using the TRIzol reagent (Invitrogen; Thermo Fisher Scientific, Inc., Waltham, MA, USA) in accordance with the manufacturer's instructions. 
Table I. Basal conditions between the experimental and control groups in the first part of the present study.

\begin{tabular}{lccc}
\hline Conditions & Y-27632 & Control & P-value \\
\hline Age (years) & $30.92 \pm 4.84$ & $30.90 \pm 4.67$ & \\
Embyo grade; n (\%) & & & \\
1 & $11(4.8)$ & $15(6.7)$ & 0.686 \\
2 & $137(60.1)$ & $120(53.8)$ & 0.178 \\
3 and 4 & $80(35.1)$ & $88(39.5)$ & 0.337 \\
Cell number; n $(\%)$ & & & \\
$2-3$ & $51(22.4)$ & $97(22.9)$ & 0.899 \\
$4-5$ & $101(44.3)$ & $74(32.6)$ & 0.864 \\
$\geq 6$ & $73(32.0)$ & $1(0.4)$ & 0.792 \\
Compact & $3(1.3)$ & 0.326
\end{tabular}

Data are expressed as the mean \pm standard deviation.

Table II. Blastocyst formation between the experimental and control groups.

\begin{tabular}{lccr}
\hline & Y-27632 $(\mathrm{n}=228)$ & Control $(\mathrm{n}=223)$ & $\mathrm{n}(\%)$ \\
$\mathrm{n}(\%)$ & 26 -value \\
\hline 5 & $26(11.4)$ & $44(19.7)$ & $0.015^{\mathrm{a}}$ \\
6 & $8(11.0)$ & $5(2.2)$ & 0.077 \\
7 & $2(0.9)$ & $1(0.4)$ & 0.422 \\
8 & $61(26.8)$ & $64(28.7)$ & 0.575 \\
Total & & 0.644 \\
\hline
\end{tabular}

${ }^{\mathrm{a}} \mathrm{P}<0.05$ between the $\mathrm{Y}-27632$ and control groups.

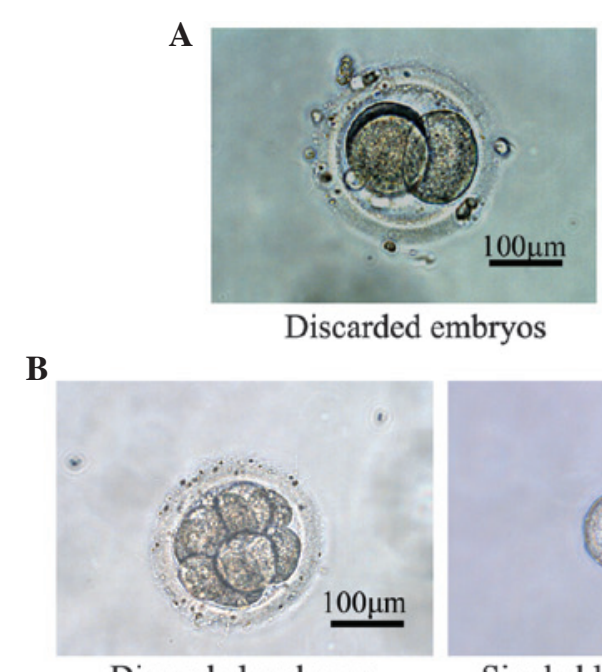

Discarded embryos

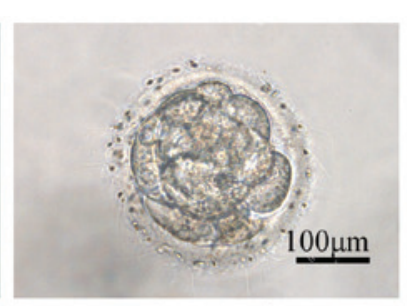

Compaction

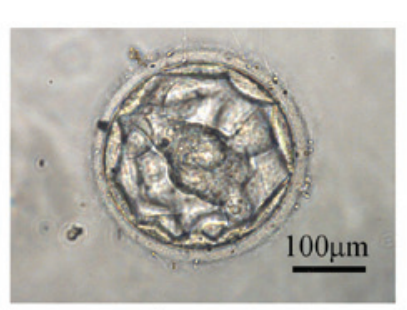

Blastocyst
Discarded embryos

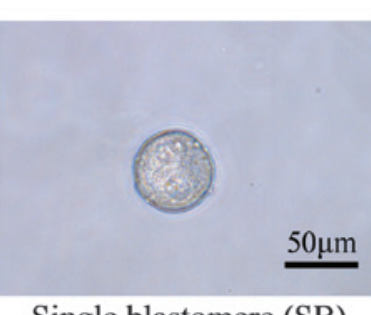

Single blastomere (SB)

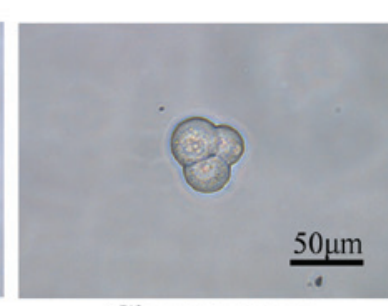

Cleavage stage

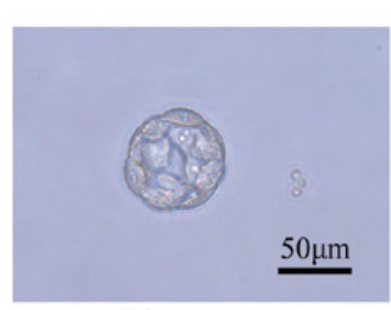

Blastocyst

Figure 1. Representative examples of blastocyst formation. (A) Representative examples of the development of discarded embryos. Day-3 poor quality embryos were collected, the cleaved embryo developed to compaction, which further developed to an expanded blastocyst. (B) Representative examples of the development of single blastomeres. Day-3 poor quality embryos were collected, single blastomeres were isolated, which developed to cleavage stage and then to blastocyst stage.

A total of $1 \mu \mathrm{g}$ total RNA was reverse transcribed using the Moloney murine leukemia virus reverse transcriptase (Promega Corp, Madison, WI, USA). Subsequently, cDNA samples were used as the template for PCR. RT-qPCR was performed using SYBR Green and an ABI 7300 Real-Time PCR System (Applied Biosystems; Thermo Fisher Scientific, 
Table III. Blastocyst grade between the experimental and control groups.

\begin{tabular}{|c|c|c|c|}
\hline Grade & $\begin{array}{c}\mathrm{Y}-27632(\mathrm{n}=26) \\
\mathrm{n}(\%)\end{array}$ & $\begin{array}{c}\text { Control }(\mathrm{n}=44) \\
\mathrm{n}(\%)\end{array}$ & P-value \\
\hline \multicolumn{4}{|c|}{ Blastocyst } \\
\hline 3 & $10(38.5)$ & $10(22.7)$ & 0.159 \\
\hline 4 & $14(53.8)$ & $28(63.6)$ & 0.419 \\
\hline 5 & $2(7.7)$ & $6(13.6)$ & 0.714 \\
\hline 6 & 0 & 0 & - \\
\hline \multicolumn{4}{|c|}{ Inner cell mass } \\
\hline A & $4(15.4)$ & $4(9.1)$ & 0.681 \\
\hline B & $9(34.6)$ & $16(36.5)$ & 0.882 \\
\hline $\mathrm{C}$ & $13(50.0)$ & $24(54.6)$ & 0.712 \\
\hline \multicolumn{4}{|c|}{ Trophectoderm } \\
\hline A & $4(15.4)$ & $10(22.7)$ & 0.458 \\
\hline $\mathrm{B}$ & $12(46.2)$ & $19(43.2)$ & 0.809 \\
\hline $\mathrm{C}$ & $10(38.5)$ & $15(34.1)$ & 0.712 \\
\hline
\end{tabular}

Grade 1, early blastocyst with blastocoel less than 50\% of the volume; grade 2, early blastocyst with blastocoel occupying 50-80\%; grade 3 , non-expanded blastocyst with large blastocoel; grade 4, expanded blastocyst; grade 5, the blastocyst has started to hatch; grade 6, the blastocyst is completely hatched. Inner cell mass grade: A, high number of tightly packed cells; B, few cells and loosely packed; C, almost no cells. Trophectoderm grade: A, high number of cells; B, few cells; C, almost no cells.

Table IV. Survival analysis of blastocyst formation time.

\begin{tabular}{lcc}
\hline Group & Median formation time (days) & P-value \\
\hline Y-27632 & 6.18 & $0.005^{\mathrm{a}}$ \\
Control & 5.73 & \\
\hline
\end{tabular}

${ }^{\mathrm{a}} \mathrm{P}<0.05$, significant difference between $\mathrm{Y}-27632$ and control groups.

Inc.) according to the manufacturer's instructions. An initial DNA denaturation step at $95^{\circ} \mathrm{C}$ for $10 \mathrm{~min}$ was followed by 40 cycles of denaturation at $95^{\circ} \mathrm{C}$ for $15 \mathrm{sec}$, primer annealing at $60^{\circ} \mathrm{C}$ for $1 \mathrm{~min}$, and an extension step at $72^{\circ} \mathrm{C}$ for $15 \mathrm{sec}$. RT-qPCR was performed in triplicate on blastocyst samples to quantify the mRNA expression of E-cadherin. The housekeeping gene, glyceraldehyde 3-phosphate dehydrogenase (GAPDH), was used as an internal control. The primer sequences were as follows: GAPDH, forward 5'-CGGAGT CAACGGATTTGGTCG-3' and reverse 5'-CCTGGAAGA TGGTGATGGG-3'); and E-cadherin, forward 5'-GGCACT TGACCCTGATACG-3' and reverse 5'-GCTGGACCGAGA GAGTTAC-3'. Data were analyzed with Sequence Detector software (Applied Biosystems; Thermo Fisher Scientific, Inc.). Relative expression of E-cadherin was calculated using the $2^{-\Delta \Delta \mathrm{Cq}}$ method.

Statistical analysis. Statistical comparisons between groups were performed using analysis of variance, $\chi^{2}$ test, Fisher's exact test, Student's $t$-test and Breslow survival analysis, using SPSS software version 13.0 (SPSS, Inc., Chicao, IL, USA). $\mathrm{P}<0.05$ was considered to indicate a statistically significant difference.
Table V. Blastocyst formation rate of human individual blastomeres.

\begin{tabular}{lcc}
\hline Stage & $\begin{array}{c}\text { Y-27632 }(\mathrm{n}=596) \\
\mathrm{n}(\%)\end{array}$ & $\begin{array}{c}\text { Control }(\mathrm{n}=596) \\
\mathrm{n}(\%)\end{array}$ \\
\hline Cleavage (day 4) & $213(35.7)$ & $203(34.1)$ \\
Blastocyst & $82(13.8)^{\mathrm{a}}$ & $51(8.6)$ \\
\hline
\end{tabular}

${ }^{\mathrm{a}} \mathrm{P}<0.004$ vs. control.

\section{Results}

In the first part of the present study (Fig. 2), 526 embryos were alternately allocated to blastocyst culture, with or without Y-27632 ( $\mathrm{n}=263$ each). Following confirmation that 75 of these 526 fertilized eggs had not cleaved, of the remaining embryos, 228 and 223 were cultured with and without Y-27632, respectively. Basal conditions, including embryonic grade, were comparable between the two groups (Table I). In total, 61 (26.8\%) and $64(28.7 \%)$ embryos of the Y-27632 and control groups reached the blastocyst stage, although this difference was not statistically significant $(\mathrm{P}=0.644$; Table II). Comparison of the day 5 blastocyst grades between the two groups revealed no statistically significant difference (Table III). The duration of blastocyst formation was also compared between the two groups. On day 5, the blastocyst formation ratio in the experimental group was $11.4 \%(26 / 228)$, which was significantly lower $(\mathrm{P}=0.015)$, compared with the corresponding rate in the control group $(19.7 \%$; 44/223; Table II). Breslow survival analysis of the blastocyst formation time was performed. The median formation time in the 


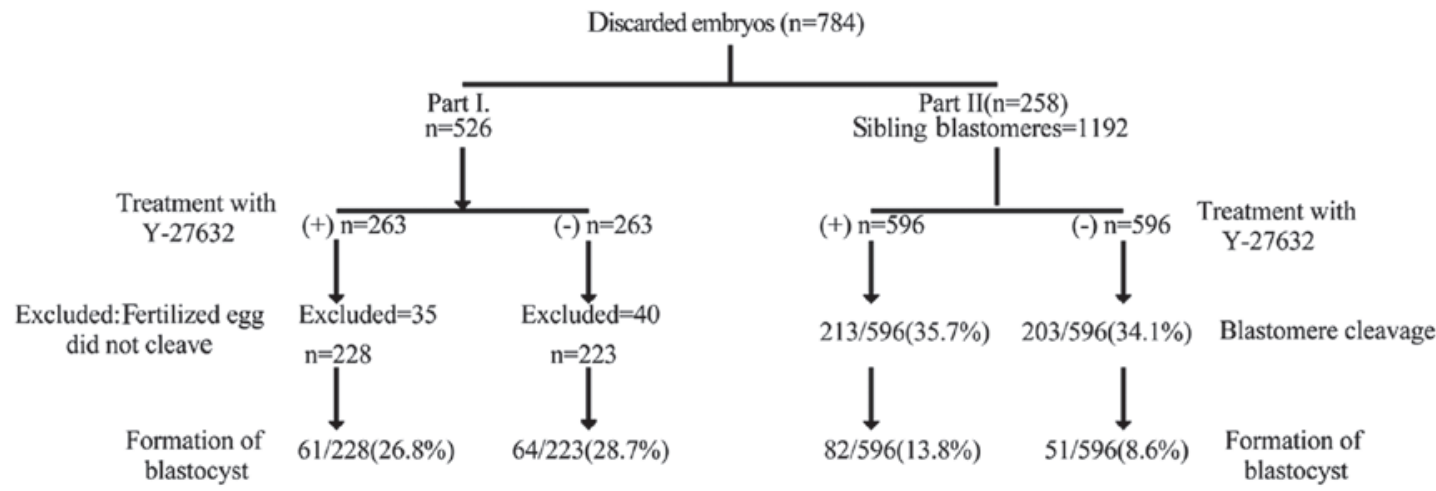

Figure 2. Experimental strategy to determine the effect of Y-27632 on discarded embryos and single blastomeres. A total of 784 discarded fresh human embryos were used. In the first part of the experiment, 526 embryos were collected and alternately allocated to blastocyst culture with, or without, Y-27632. In the second part of the experiment, 258 embryos were collected and used for blastomere isolation, and 1,192 sibling blastomeres of similar size were paired and allocated to blastocyst culture with, or without, Y-27632. Blastocyst formation and blastomere development were recorded.

A

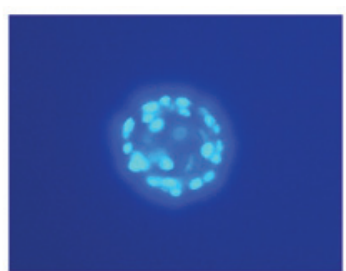

Cell calculation

C

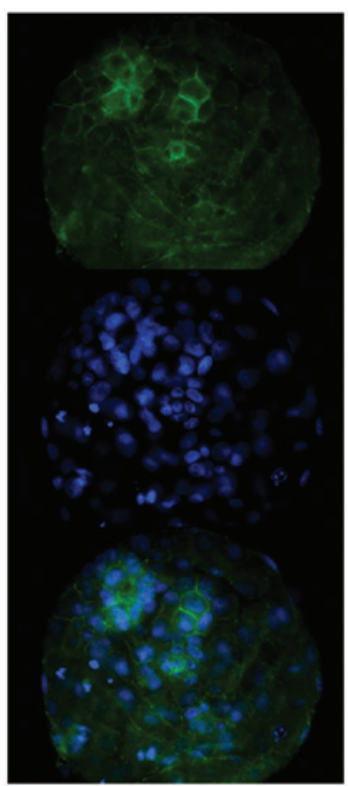

Control
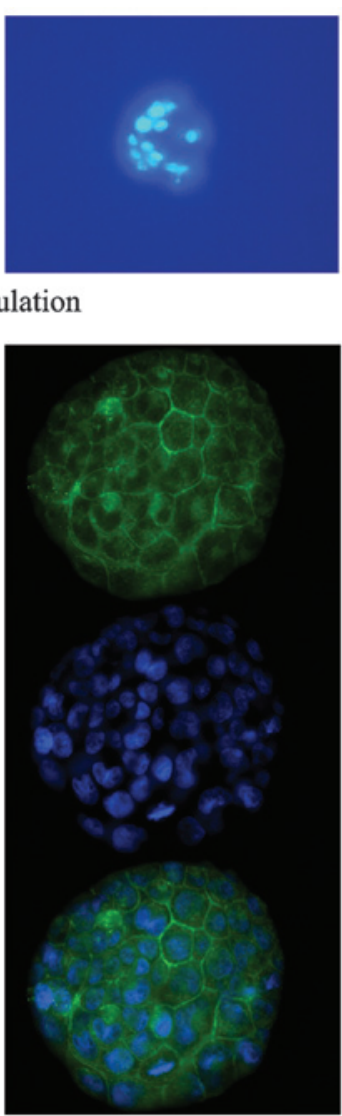

Y-27632
B

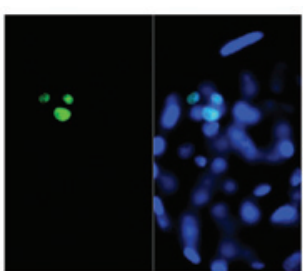

OCT3/4(+)

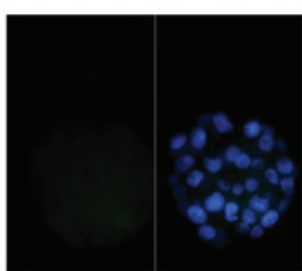

OCT3/4(-)

D

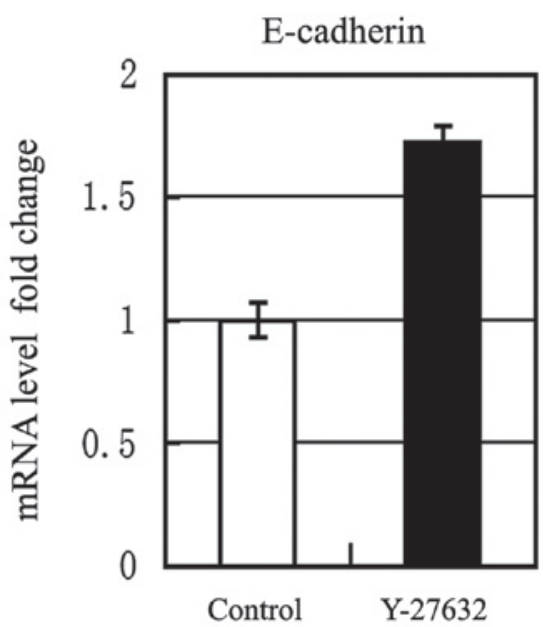

Figure 3. Effect of Y-27632 on single blastomeres. (A) Nuclei were counterstained with Hoechst-33342 to count the number of cells. (B) Representative examples of immunocytochemical staining against OCT3/4. (C) Representative examples of the protein expression levels of E-cadherin, determined using immunofluorescence in the experimental group and control group. The three images of each group were E-cadherin expression alone (green), stained with DAPI alone (blue) and the combined image. Culture of blastomeres in the presence of $10 \mu \mathrm{M}$ Y-27632 resulted in upregulation of the expression of E-cadherin. (D) Effects of Y-27632 on the mRNA expression of E-cadherin. E-cadherin mRNA levels were measured using reverse transcription-quantitative polymerase chain reaction analysis. Quantitative results were standardized to the levels of GAPDH and presented as the fold change over the control group ( $\mathrm{P}=0.22$, vs. control). OCT3/4, octamer-binding transcription factor.

experimental group was 6.18 days, which was significantly higher than that of the control group (5.73 days; $\mathrm{P}=0.005$; Table IV).

Following confirmation that Y-27632 increased the ratio of formation of blastocysts from single human blastomeres, the present study obtained 1,192 blastomeres from 258 discarded day 3 embryos, and sibling blastomeres of similar sizes were equally allocated into experimental and control groups ( $\mathrm{n}=596$ each; Fig. 1). On day 4, 213 (35.7\%) blastomeres in the Y-27632 group, and 203 (34.1\%) blastomeres in the control group had cleaved to form two or more cells; however, no statistically significant difference was observed 
in the number of cleaved cells between the two groups $(\mathrm{P}=0.543$; Table $\mathrm{V})$.

Treatment with Y-27632 increased the blastocyst formation ratio of human individual blastomeres. The results revealed that 82 blastocysts of 596 blastomeres (13.8\%) and 51 blastocysts of 596 blastomeres $(8.6 \%)$ were formed in the presence and absence of Y-27632, respectively, and this difference was statistically significant between the two groups $(\mathrm{P}=0.004$; Table V).

On day 6, a total of 33 and 14 blastocysts in the experimental and control groups, respectively, were stained to count the cell number. The average numbers of blastocysts were $24.7 \pm 13.3$ and $24.6 \pm 8.8$, in the presence and absence of Y-27632, respectively. However, this difference was not statistically significant $(\mathrm{P}=0.973$; Fig. 3A).

On day 7, the expression of Oct3/4 was detected in 13 and 8 blastocysts in the experimental and control groups, respectively. Only a small percentage of the blastocysts expressed Oct $3 / 4$, and the number of cells, which stained positive for Oct $3 / 4$ ranged between 2 and 5 per blastocyst. The Oct3/4(+) blastocyst rates were $30.8 \%(4 / 13)$ and $25.0 \%(2 / 8)$ in the experimental and control groups, respectively, which was not a statistically significant difference ( $\mathrm{P}=0.776$; Fig. 3B).

The protein expression of E-cadherin was detected in eight blastocysts in both the experimental and control groups using indirect immunofluorescence. The culture of blastocysts in the presence of Y-27632 resulted in an upregulation in the expression of E-cadherin (Fig. 3C). The mRNA expression of E-cadherin was detected in 20 blastocysts in both the experimental and control groups using RT-qPCR. There was a significant increase in the expression of E-cadherin in the Y-27632 group ( $\mathrm{P}=0.022$; Fig. 3D).

\section{Discussion}

The present study initially investigated the effect of the ROCK inhibitor, Y-27632, on fresh human cleavage-stage embryos, and on the development of single blastomeres. The results of these investigations demonstrated that Y-27632 increased the ratio of formation of blastocysts from single human blastomeres. However, Y-27632 inhibited the formation of day 5 blastocysts and delayed the total formation of blastocysts from discarded human embryos.

In contrast to previous reports, the data of the present study suggested that Y-27632 did not affect the total ratio of blastocyst formation or the quality of the human embryos. Cortes et al reported that Y-27632 increases post-thaw embryo survival and development up to the blastocyst stage (14). However, in the experiments in the present study used discarded fresh embryos from fresh IVF-ET cycles. This suggests that Y-27632 may have a positive effect on post-thaw embryos, rather than on fresh embryos. This is similar to another study, which reported that Y-27632 improves the revivability of bovine blastocysts following vitrification and warming (13). By contrast, other studies have suggested that ROCK inhibition prevents early mouse embryo development and blastocyst cavity formation $(12,17,26)$, and in these studies it was concluded that ROCK is involved in early embryonic development. However, in these previous studies, the precise time of blastocyst formation was not recorded. In the present study, the precise time of blastocyst formation was recorded every day. On day 5, the blastocyst formation ratio of the embryos in the Y-27632 group was lower than that in the control group, and this difference was statistically significant. The survival analysis in the present study indicated that ROCK inhibition delayed the formation of blastocysts by almost half a day. Thus, the results of the present study led to the hypothesis that Y-27632 negatively affects fresh human embryos by delaying the time of development. However, due to the ethical issues associated with experimentation on human embryos, no further investigations on clinical embryo transfer trials can be performed to prove the conclusions of the present study.

To further investigate the involvement of ROCK in blastomere development, the present study investigated the effect of Y-27632 on single blastomeres. Of note, Y-27632 increased the blastocyst formation ratio of single blastomeres in the discarded human embryos. Taei et al found that, when combined with the pluripotency-enhancing small molecule, CHIR99021, Y-27632 enhanced the generation of hESCs from single blastomeres of fair and poor-quality embryos (29). Other studies have also demonstrated the roles of Y-27632 in pluripotent cells, including iPS cells and hESCs. In addition, Y-27632 improves the survival of dissociative hESCs and cryopreserved single hESCs. It has been suggested that ROCK inhibition increases the survival of hESCs as a result of increased cell-cell interactions, reduced dissociation-induced apoptosis, maintenance of pluripotent characteristics and enhanced cell propagation. In the present study, the expression level of OCT3/4 express did not differ significantly between the experimental and control groups. Therefore, it was concluded that Y-27632 had no effect on maintaining pluripotent characteristics during the development of single blastomeres. Furthermore, the present study counted the number of blastocysts, and found that Y-27632 had no effect on cell propagation. However, the results of the experiment confirmed that Y-27632 enhanced the expression of E-cadherin at the protein and mRNA levels. Cell-cell connections were improved by Y-27632, which may be one of mechanisms by which Y-27632 increases the blastocyst formation ratio of single blastomeres. However, the mechanism is complex and further investigations are required to clarify this. However, based on the limited sample size in the present study, no further experiments were performed to investigate the mechanism in detail.

Notably, Y-27632 had different effects on the cleavage-stage embryo and single blastomeres. Several previous animal studies have demonstrated that Y-27632 has a negative effect on embryonic development $(12,17,26)$. Y-27632 suppressed embryo cleavage, compaction and blastocyst formation, and these results suggest that ROCK is involved in polarization, cleavage and blastocoel cavity formation in early cleavage-stage embryos. In the present study, the inhibition of ROCK by Y-27632 resulted in delayed blastocyst formation. The possible underlying mechanism may be that interblastomere adhesion was enhanced by Y-27632, and different blastomeres with different fates were tightly bound together, leading to low efficiency of development. In contrast to the results with whole embryos, single blastomeres cultured with Y-27632 produced more blastocysts. The possible underlying mechanism for this many be that Y-27632 enhanced cell-cell adhesion and 
markedly reduced dissociation-induced apoptosis. Y-27632 has already been shown to permit the survival of dissociated pluripotent cells, including iPS cells and hESCs. However, the different effects of Y-27632 on cleavage-stage embryos and single blastomeres observed in the present study, together with the results of previous studies, indicate that ROCK has different roles in embryos of different species, and at different developmental stages. The mechanism is complex and more well-designed experiments are required for clarification.

In conclusion, the present study demonstrated that Y-27632 increased the rate of formation of blastocysts from single human blastomeres, but delayed the formation of blastocysts from discarded human embryos. Further studies are required to investigate the various roles of ROCK inhibition on the development of early human embryos and blastomeres, such as how this cytokine may be interacting with additional regulatory molecules.

\section{Acknowledgements}

This study was supported by grants from the Key Laboratory of Guangdong Province and the National Natural Science Foundation of China (grant nos. 81100472 and 81270750).

\section{References}

1. Sutcliffe AG and Ludwig M: Outcome of assisted reproduction. Lancet 370: 351-359, 2007.

2. Mantikou E, Youssef MA, van Wely M, van der Veen F, Al-Inany HG, Repping S and Mastenbroek S: Embryo culture media and IVF/ICSI success rates: a systematic review. Hum Reprod Update 19: 210-220, 2013.

3. Bontekoe S, Blake D, Heineman MJ, Williams EC and Johnson N: Adherence compounds in embryo transfer media for assisted reproductive technologies. Cochrane Database Syst Rev 7: CD007421, 2010.

4. Carney SK, Das S, Blake D, Farquhar C, Seif MM and Nelson L: Assisted hatching on assisted conception (in vitro fertilization (IVF) and intracytoplasmic sperm injection (ICSI). Cochrane Database Syst Rev 12: CD001894, 2012.

5. Bontekoe S, Mantikou E, van Wely M, Seshadri S, Repping S and Mastenbroek S: Low oxygen concentrations for embryo culture in assisted reproductive technologies. Cochrane Database Syst Rev 7: CD008950, 2012.

6. Watson AJ and Barcroft LC: Regulation of blastocyst formation. Front Biosci 6: D708-D730, 2001.

7. Dard N, Breuer M, Maro B and Louvet-Vallée S: Morphogenesis of the mammalian blastocyst. Mol Cell Endocrinol 282: 70-77, 2008.

8. Gumus E, Bulut HE and Kaloglu C: Cytoskeletal changes in oocytes and early embryos during in vitro fertilization process in mice. Anat Histol Embryol 39: 51-58, 2010.

9. Rawe VY, Payne C and Schatten G: Profilin and actin-related proteins regulate microfilament dynamics during early mammalian embryogenesis. Hum Reprod 21: 1143-1153, 2006.

10. Ishizaki T, Uehata M, Tamechika I, Keel J, Nonomura K, Maekawa M and Narumiya S: Pharmacological properties of Y-27632, a specific inhibitor of rho-associated kinases. Mol Pharmacol 57: 976-983, 2000.

11. Kawagishi R, Tahara M, Sawada K, Ikebuchi Y, Morishige K, Sakata M, Tasaka K and Murata Y: Rho-kinase is involved in mouse blastocyst cavity formation. Biochem Biophys Res Commun 319: 643-648, 2004.

12. Duan X, Chen KL, Zhang Y, Cui XS, Kim NH and Sun SC: ROCK inhibition prevents early mouse embryo development. Histochem Cell Biol 142: 227-233, 2014.
13. Hochi S, Abdalla H, Hara H, Shimoda M, Morita H, Kuwayama $M$ and Hirabayashi M: Stimulatory effect of Rho-associated coiled-coil kinase (ROCK) inhibitor on revivability of in vitro-produced bovine blastocysts after vitrification. Theriogenology 73: 1139-1145, 2010.

14. Cortes JL, Sanchez L, Ligero G, Gutierrez-Aranda I, Catalina P, Elosua C, Leone PE, Montes R, Bueno C, Ramos-Mejía V, et al: Mesenchymal stem cells facilitate the derivation of human embryonic stem cells from cryopreserved poor-quality embryos. Hum Reprod 24: 1844-1851, 2009.

15. Clayton L, Hall A and Johnson MH: A role for Rho-like GTPases in the polarisation of mouse eight-cell blastomeres. Dev Biol 205: 322-331, 1999.

16. Koike S, Keino-Masu K and Masu M: Deficiency of autotaxin/lysophospholipase D results in head cavity formation in mouse embryos through the LPA receptor-Rho-ROCK pathway. Biochem Biophys Res Commun 400: 66-71, 2010.

17. Laeno AM, Tamashiro DA and Alarcon VB: Rho-associated kinase activity is required for proper morphogenesis of the inner cell mass in the mouse blastocyst. Biol Reprod 89: 122, 2013.

18. Geens M, Mateizel I, Sermon K, De Rycke M, Spits C, Cauffman G, Devroey P, Tournaye H, Liebaers I and Van de Velde H: Human embryonic stem cell lines derived from single blastomeres of two 4-cell stage embryos. Hum Reprod 24: 2709-2717, 2009.

19. Van de Velde H, Cauffman G, Tournaye H, Devroey P and Liebaers I: The four blastomeres of a 4-cell stage human embryo are able to develop individually into blastocysts with inner cell mass and trophectoderm. Hum Reprod 23: 1742-1747, 2008.

20. Watanabe K, Ueno M, Kamiya D, Nishiyama A, Matsumura M, Wataya T, Takahashi JB, Nishikawa S, Nishikawa S, Muguruma K and Sasai Y: A ROCK inhibitor permits survival of dissociated human embryonic stem cells. Nat Biotechnol 25: 681-686, 2007.

21. Pakzad M, Totonchi M, Taei A, Seifinejad A, Hassani SN and Baharvand H: Presence of a ROCK inhibitor in extracellular matrix supports more undifferentiated growth of feeder-free human embryonic and induced pluripotent stem cells upon passaging. Stem Cell Rev 6: 96-107, 2010.

22. Rizzino A: Stimulating progress in regenerative medicine: Improving the cloning and recovery of cryopreserved human pluripotent stem cells with ROCK inhibitors. Regen Med 5: 799-807, 2010.

23. Zhang P, Wu X, Hu C, Wang P and Li X: Rho kinase inhibitor Y-27632 and Accutase dramatically increase mouse embryonic stem cell derivation. In Vitro Cell Dev Biol Anim 48: 30-36, 2012.

24. Horiguchi A, Yazaki K, Aoyagi M, Ohnuki Y and Kurosawa H: Effective Rho-associated protein kinase inhibitor treatment to dissociate human iPS cells for suspension culture to form embryoid body-like cell aggregates. J Biosci Bioeng 118: 588-592, 2014.

25. Sivasubramaiyan K, Totey S, Bhat V, Totey SM and Deb K: Y-27632 enhances differentiation of blastocyst like cystic human embryoid bodies to endocrinologically active trophoblast cells on a biomimetic platform. J Biomed Sci 16: 88, 2009.

26. Zhang Y, Duan X, Xiong B, Cui XS, Kim NH, Rui R and Sun SC: ROCK inhibitor Y-27632 prevents porcine oocyte maturation. Theriogenology 82: 49-56, 2014.

27. Steer CV, Mills CL, Tan SL, Campbell S and Edwards RG: The cumulative embryo score: A predictive embryo scoring technique to select the optimal number of embryos to transfer in an in-vitro fertilization and embryo transfer programme. Hum Reprod 7: 117-119, 1992.

28. Gardner DK, Lane M and Schoolcraft WB: Culture and transfer of viable blastocysts: A feasible proposition for human IVF. Hum Reprod 15 (Suppl 6): S9-S23, 2000.

29. Taei A, Hassani SN, Eftekhari-Yazdi P, Rezazadeh Valojerdi M, Nokhbatolfoghahai M, Masoudi NS, Pakzad M, Gourabi H and Baharvand $\mathrm{H}$ : Enhanced generation of human embryonic stem cells from single blastomeres of fair and poor-quality cleavage embryos via inhibition of glycogen synthase kinase $\beta$ and Rho-associated kinase signaling. Hum Reprod 28: 2661-2671, 2013. 